\title{
Control and Resolution Mechanisms of the Inflammatory Response 2016
}

\author{
Víctor M. Baizabal-Aguirre, ${ }^{1}$ Carlos Rosales, ${ }^{2}$ \\ Constantino López-Macías, ${ }^{3,4}$ and Marisa I. Gómez ${ }^{5}$ \\ ${ }^{1}$ Centro Multidisciplinario de Estudios en Biotecnología, Facultad de Medicina Veterinaria y Zootecnia, Universidad Michoacana de \\ San Nicolás de Hidalgo, Km. 9.5 Carretera Morelia-Zinapécuaro S/N, 58893 Morelia, MICH, Mexico \\ ${ }^{2}$ Departamento de Inmunología, Instituto de Investigaciones Biomédicas, Universidad Nacional Autónoma de México, \\ Apdo. Postal 70228, Ciudad Universitaria, 04510 Ciudad de México, Mexico \\ ${ }^{3}$ Unidad de Investigación Médica en Inmunoquímica, Hospital de Especialidades del Centro Médico Nacional Siglo XXI, \\ Instituto Mexicano del Seguro Social, Avenida Cuauhtémoc 330 Col. Doctores, 06170 Ciudad de México, Mexico \\ ${ }^{4}$ Nuffield Department of Medicine, Peter Medawar Building for Pathogen Research, University of Oxford, South Parks Rd., \\ Oxford OX1 3SY, UK \\ ${ }^{5}$ Instituto de Investigaciones en Microbiología y Parasitología Médica (IMPaM), Universidad de Buenos Aires, Consejo Nacional para \\ la Promoción de la Ciencia y la Tecnología, Paraguay 2155 Piso 12, 1121 Ciudad Autónoma de Buenos Aires, Argentina
}

Correspondence should be addressed to Víctor M. Baizabal-Aguirre; baizabal@umich.mx

Received 8 December 2016; Accepted 8 December 2016

Copyright ( 2016 Víctor M. Baizabal-Aguirre et al. This is an open access article distributed under the Creative Commons Attribution License, which permits unrestricted use, distribution, and reproduction in any medium, provided the original work is properly cited.

In the last 20 years the field of the innate immunity has advanced notably since the pioneering work on the discovery of Toll-like receptors. A considerable number of studies on the molecular mechanisms triggering the inflammatory response have been described. A search in PubMed with the words "inflammatory response" for the current year gives 11,000 articles, which means an approximate 917 articles per month and 30 per day. This amount of information reflects the interest of the scientific community not only to understand the essential mechanisms involved in the inflammatory response but also to translate this knowledge for the treatment of chronic and degenerative human diseases. Now that the main participating molecules and signaling transduction mechanisms activated during the inflammatory response have been established, researchers have begun to elucidate how a tissue is able to control/resolve inflammation and regain homeostasis. This is because a failure of the mechanisms that self-regulate and resolve the inflammatory process may lead to chronic inflammation, and this in turn may cause degenerative diseases such as cancer, diabetes, and autoimmune and cardiovascular diseases. Although many investigations have described synthetic or natural molecules that inhibit inflammation, no clear picture on the control and resolution mechanisms have emerged. This special issue is an attempt to contribute to our knowledge of the molecular programs used by the cell to control and resolve inflammation. We hope this issue may be a good reference to all interested in the complex process of the inflammation control and resolution.

Inflammation is dictated by the coordinated action of many soluble mediators and effector cells. TNF- $\alpha$ is a major player in this process as this cytokine is able to act on multiple cell types. In this issue, in the context of a clinically relevant entity such as asthma, J. ReyesGarcía et al. have explored the effects of TNF- $\alpha$ on tracheal smooth muscle cells and demonstrated that it inhibits LType $\mathrm{Ca}^{2+}$ channels through a ERK $1 / 2$ pathway leading to the decrease in voltage dependent inward calcium current. They propose that this could be a compensatory mechanism during hyperresponsiveness of the airways. In the context of airway inflammation, the contribution of G. D. Albano et al. has provided evidence for the biological function of hyaluronan in regulating inflammation, using an in vitro model of nasal inflammation mediated by IL-17A. They 
dissected the molecular mechanisms by which high molecular weight hyaluronan induces anti-inflammatory responses and provided evidence for the potential of this molecule as coadjuvant of the classic anti-inflammatory/antioxidative treatment of nasal epithelial cells during inflammation. In line with these studies on mucosal inflammation, C. Bernardazzi et al. have reviewed the literature about neuroimmunomodulation in the gut focusing on inflammatory bowel disease. In addition to the interplay between the nervous system and the immune system, they discussed the role of the microbiota in the regulation of the neuroimmune crosstalk involved in intestinal homeostasis and inflammation. Moving into inflammation induced during infectious diseases, M. Molteni et al. reviewed the role of Toll-Like Receptor 4 in infectious and noninfectious inflammation. They focused on the research progress during the last years about the effects of TLR4 signaling in pathological conditions.

Because inflammation has been involved in the pathogenesis of multiple diseases it is therefore important to understand the mechanisms involved in the pathogenic effects of the inflammatory responses as well as to explore different therapeutic strategies. Accordingly, G. D. Duerr et al. reported the identification of important mechanisms involved in the cardioprotective effects on metallothioneins MT1 and MT2 in a murine model of brief repetitive ischemia and reperfusion, providing valuable information that could lead to novel strategies for prevention or treatment of ischemic heart disease. E. van der Gracht et al. reviewed the current knowledge about the contribution of innate lymphoid cells in the repairing process of the epithelial barrier and tissue homeostasis and the association of these mechanisms with the control of inflammatory bowel disease. F. Capone et al. reviewed the use of the "omics" approach to evaluate the cytokine expression patterns in patients with cancer as a tool to define new strategies for diagnostics and prognostics. H.-P. Wu et al. analysed the IL-12 production and HLA-DR expression on cells from severe septic shock patients treated with low-dose steroids, providing important information that could help in the discussion about this treatment. V. Carregaro et al. reported the use of 15-deoxy $\Delta^{12,14}$-prostaglandin J2, to protect mice from disease aggravation in an arthritis experimental model and identify some mechanisms contributing to this process that involves several CD4 T cell populations.

Pro- and anti-inflammatory cytokines are crucial to trigger and control the inflammatory response, respectively. In this special issue $\mathrm{M}$. Bigoni et al. reported the concentrations of selected pro- and anti-inflammatory cytokines in knee joints with anterior cruciate ligament injuries. In patients with this type of lesions, the reconstruction of the ligament does not decrease the incidence of posttraumatic osteoarthritis, indicating that a strong inflammatory state develops after surgery. The levels of IL-6, IL-8, and IL-10 are elevated just after knee injury and returned to almost normal levels after one month and prior to surgery, but they increased again considerably one month after reconstruction of the ligament. Thus, although the knee recovers its function, the inflammation that develops later causes damage to the bone. These findings suggest that, in addition to surgery, control of inflammation has to be considered as part of the therapy for knee injuries. Similarly, in search for new therapeutic targets for controlling inflammation, K. L. McCoy reviewed the interaction between the endocannabinoid system and the Tolllike receptor family. Because innate immune cells express cannabinoid receptors and produce endogenous cannabinoids and, in turn, the endocannabinoid system modulates local inflammatory responses, understanding their crosstalk would help fulfill the promise of cannabinoids as therapeutic agents. In line with this study, A. Janus et al. described how insulin resistance and endothelial dysfunction are connected to each other by numerous metabolic pathways and reviewed the common therapeutic targets for insulin resistance, endothelial dysfunction, and vascular inflammatory reaction at a molecular level. This helps explain the multiple effects observed with drugs currently used in management of cardiovascular disease, metabolic syndrome, and diabetes.

Toll-like receptors (TLRs) play a predominant role as pattern recognition receptors that recognize conserved molecular structures present in a wide array of microbes. The original work by Y. Qiu et al. presented evidence indicating that mice TLR $2^{-l-}$ contained a reduced number of intraepithelial type b lymphocytes (bIEL). They found that the small amount of bIEL remaining did not proliferate actively and secreted less IL-15 than normal in the intestinal mucosa. These authors concluded that lack of TLR2 is one of the factors conferring on mice a high susceptibility to dextran sulfate sodiuminduced colitis. Periodontal diseases, particularly chronic periodontitis caused by Porphyromonas gingivalis, are one of the most common inflammatory conditions found in human beings. In the review article by P. C. Carvalho-Filho et al. the role of $P$. gingivalis in the immunopathogenesis of chronic periodontitis is clearly explained. Authors paid special emphasis on the properties of the virulence factor HmuY and the potential therapeutic strategies directed against it to reduce the inflammation caused by this pathogen. Finally, an interesting topic on the function of inflammation to restore tissue homeostasis was included as a review article by O. P. Kulkarni et al. They concisely explain the important function of different mediators of inflammation and how these mediators modify the environment to resolve inflammation. Importantly, these authors clearly point out that persistent inflammation may lead to tissue remodeling and fibrosis instead of regeneration after injury.

Víctor M. Baizabal-Aguirre Carlos Rosales

Constantino López-Macías Marisa I. Gómez 


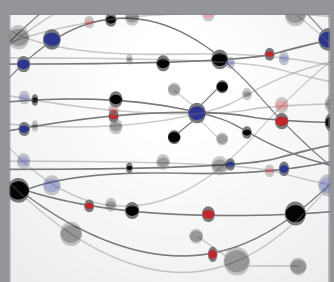

The Scientific World Journal
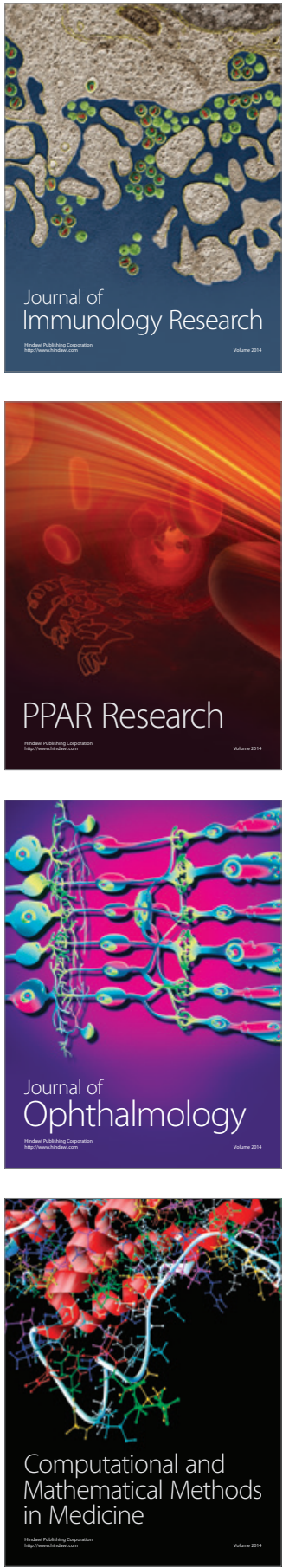

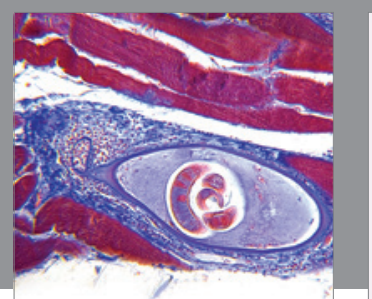

Gastroenterology Research and Practice

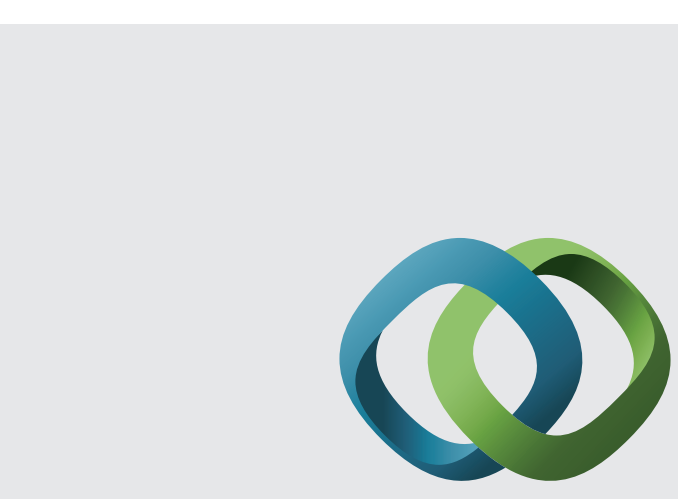

\section{Hindawi}

Submit your manuscripts at

http://www.hindawi.com
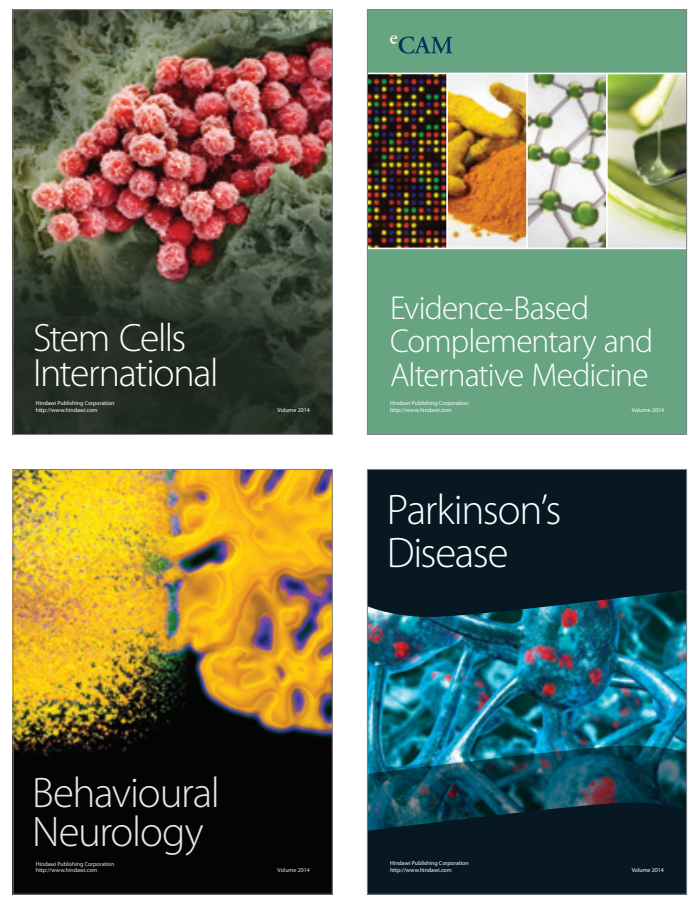
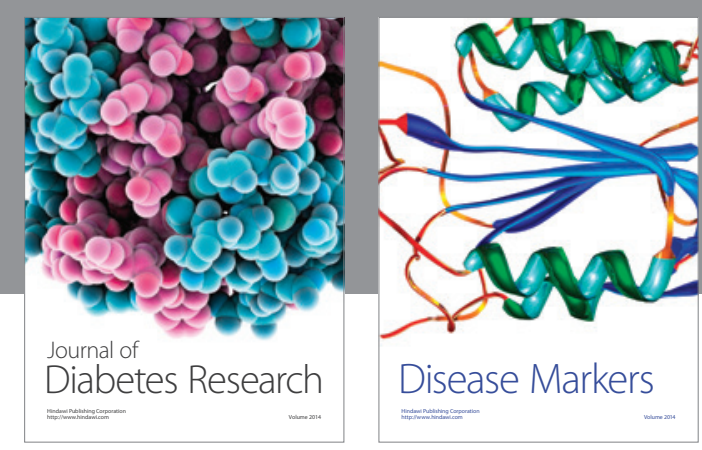

Disease Markers
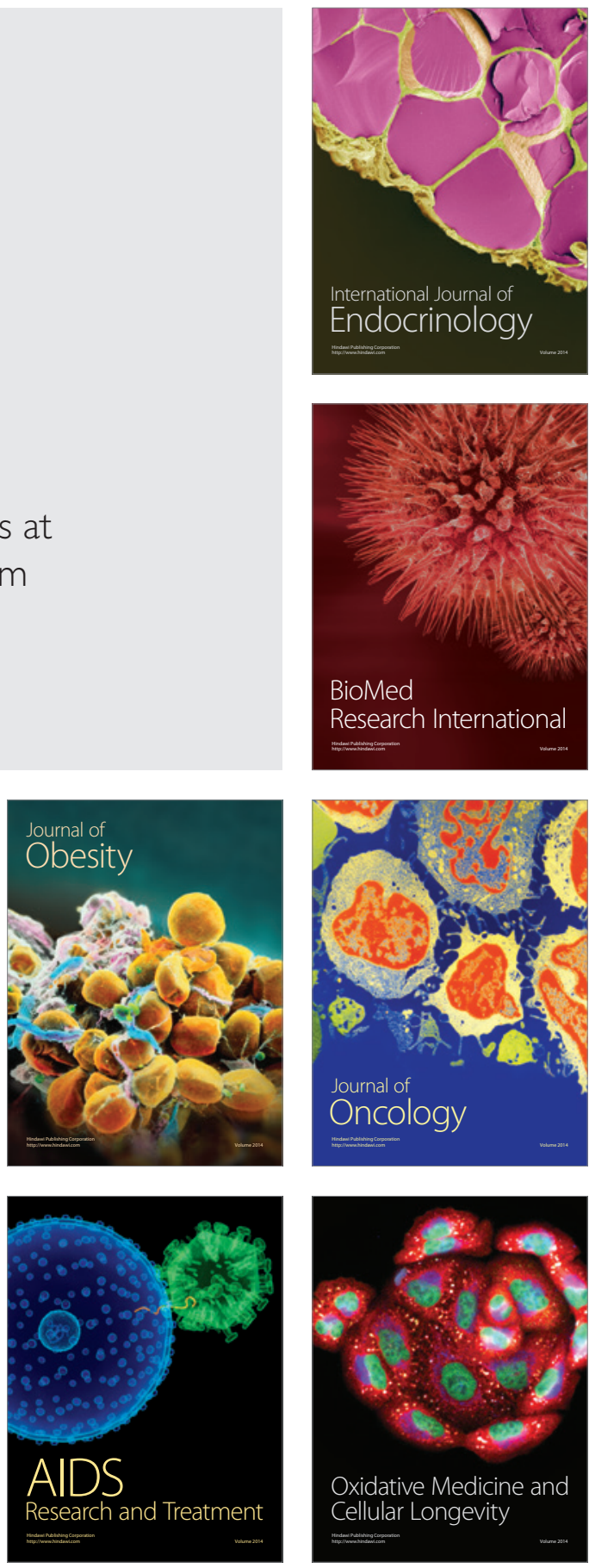